\title{
De la réflexivité du sujet aux enquêtes pratiques dans l'activité d'éducateurs spécialisés
}

From the reflexivity of the subject to the inquiry process in the activity of special educators

Sylvie Mezzena, Kim Stroumza, Laurence Seferdjeli et Pascal

\section{Baumgartner}

\section{OpenEdition}

\section{Journals}

Édition électronique

URL : http://journals.openedition.org/activites/799

DOI : 10.4000 /activites.799

ISSN : 1765-2723

Éditeur

ARPACT - Association Recherches et Pratiques sur les ACTivités

Référence électronique

Sylvie Mezzena, Kim Stroumza, Laurence Seferdjeli et Pascal Baumgartner, « De la réflexivité du sujet aux enquêtes pratiques dans l'activité d'éducateurs spécialisés ", Activités [En ligne], 10-2 | Octobre 2013, mis en ligne le 15 octobre 2013, consulté le 01 mai 2019. URL : http://journals.openedition.org/ activites/799; DOI : 10.4000/activites.799

\section{c) (i)}

Activités est mis à disposition selon les termes de la licence Creative Commons Attribution - Pas d'Utilisation Commerciale - Pas de Modification 4.0 International. 


\title{
De la réflexivité du sujet aux enquêtes pratiques dans l'activité d'éducateurs spécialisés
}

\author{
Sylvie Mezzena
}

Haute École de Travail Social/Hı S-G1 , sylvie.mezzena@hesge.ch

Kim Stroumza

Haute École de Travail Social /H1 S-G1, kim.stroumza@hesge.ch

Laurence Seferdjeli

Haute École de Santé/H1 S-G1 , Laurence.seferdjeli@hesge.ch

Pascal Baumgartner ${ }^{1}$

Rélisateur indépendant, http://www.ikonprod.ch - pb@ikonprod.ch

\begin{abstract}
From the reflexivity of the subject to the inquiry process in the activity of special educators. Drawing on Ogien and Quere's situated approach to French sociology of action, linked to Dewey's pragmatist philosophy, our analysis of the activities of special educators combined with a broader literature on work analysis suggests that the reflexivity of professionals is insufficient to account for the construction and effectiveness of the intervention process. As defined by Schon's reflexive practitioner model and its offshoots, it is a work of distancing, analyzing and formalizing their practice and as such feeds a negative and restricted conception of professionalism. Our research was conducted by a team in a shelter for adolescents from dysfunctional families, required by policy to develop a new mission of integration. Despite team meetings targeting the development of new rules, weekly seminars aimed at solving recent practical problems, informal exchanges between colleagues during the course of their work, pre-or postactivity interviews and self-analysis undertaken with professionals for research purposes, the activity continually escapes formalization. Drawing on an alternative model of professionalism based on a Deweyan notion of survey, our analysis of surveys of professional practice, both longitudinal and cross-sectional, shows that intervention requires know-how built in to the activity, even where so-called "basic" practices are concerned. It continually requires exploratory adjustments driven by the elements of the action. This suggests that the implementation of the mission does not depend on applicationist logic. This approach leads to a form of externalization of thought during the activity, defining practice as the place where knowledge is constructed without subordinating it to reflexivity as an intellectual process added to practice.
\end{abstract}

\footnotetext{
Pascal Baumgartner est réalisateur indépendant (http://www.ikon-productions.ch). Il a participé aux côtés des trois autres chercheuses comme assistant dans la recherche «La réflexivité dans l'activité des travailleurs sociaux : enjeux pour la professionnalisation » (requérante principale Sylvie Mezzena) dont il est question dans cet article. Cette recherche a été financée de 2010 à 2012 par les fonds stratégiques de la Haute école spécialisée de Suisse occidentale (H1 S-SO : http://www.hes-so.ch).
} 


\section{K1 Y WORDS}

activity, practical inquiry, dynamic combinations, perspective, externalization of thought

\section{Introduction}

Dans la professionnalisation des travailleurs sociaux, la réflexivité du sujet comme principale voire unique figure de professionnalité depuis le modèle du praticien réflexif de Schön ou ses dérivés, est tellement naturalisée non seulement dans les pratiques des formateurs, mais aussi plus largement dans les recherches, qu'elle en est devenue évidente Mezzena, 2011). Cette notion, définie par Schön comme un travail de mise à distance, de réflexion et de représentation opératoire de l'action, s'est largement diffusée dans les métiers de l'humain que sont les métiers du social, de l'enseignement ou des soins (Recherche et Formation, 2001). Un parti pris fréquent, presque systématique, fait de la réflexivité du sujet la principale voire l'unique source de guidage de l'activité. 1 lle est ainsi très influente en formation professionnelle depuis ce que Schön lui-même a appelé « le tournant réflexif » et que Tardif, Borges et Malo qualifient de «virage quasi paradigmatique » (2012). Pourtant, dans le champ de l'analyse du travail (par exemple Dejours, 1993 pour la psychodynamique du travail) et le courant de l'action située (y compris la théorie du cours d'action : Theureau, 2006), la conception de l'action et de l'intelligence sous-jacente à ce modèle a reçu de nombreuses critiques : par exemple dualisme entre pensée et action, réduction de l'exercice de l'intelligence à de l'intellect, limitation de l'action intelligente à ce qui peut être formalisé et déni d'une intelligence sous forme incarnée... De plus, ce modèle a des conséquences pour la pratique et la professionnalité dans le champ du travail social (Couturier, 2000) qui méritent de le requestionner.

Cet article se propose ainsi de reprendre les critiques qui ont pu lui être opposées et de développer dans une autre direction un modèle de la professionnalité à partir des travaux de Dewey. Schön s'est lui-même inspiré de la théorie de l'enquête de Dewey en la tirant sur un versant mentaliste logeant au final chez les professionnels le guidage de l'activité ${ }^{2}$ Nous utilisons quant à nous la notion d'enquête pratique de manière plus radicale sans la séparer de celle du partenariat avec l'environnement si cher à Dewey. Ce faisant, la détermination de l'action est distribuée sur les rapports dynamiques avec l'environnement et non pas attribuée aux seuls professionnels. Cet usage qui externalise la pensée dans l'action nous paraît plus juste non seulement pour des raisons empiriques et théoriques, mais aussi en termes de conséquences politiques pour la reconnaissance du savoir-faire des éducateurs.

Pour ce faire, nous examinerons comment une éducatrice d'un foyer accueillant des adolescents en rupture familiale et/ou sociale demande à un jeune de quitter son ordinateur pour aller ranger sa chambre. Nous tenterons de montrer le savoir-faire déployé par la professionnelle à cette occasion, sans réduire celui-ci à une réflexion intellectuelle, mais sans non plus en déduire que dès lors ce savoir-faire n'est pas intelligent. Reconstruire le savoirfaire dans une activité de base, a priori triviale et banale, est un enjeu important pour la professionnalisation des travailleurs sociaux de niveau universitaire (aussi qualifié de tertiaire). Le risque, si l'on reste sur une définition strictement réflexive de la professionnalité, est en effet de considérer que les tâches quotidiennes de contact avec les usagers, qui font le ciment du métier éducatif, ne devraient être confiées qu'à des personnes porteuses d'un diplôme de niveau inférieur sous prétexte qu'il n'y a pas suffisamment d'expertise (entendez de réflexivité) qui s'y déploie. La notion d'enquête pratique développée par Dewey offre une voie pour observer ce savoir-faire qui ne relève pas (ou pas toujours) d'une réflexivité, en étudiant l'activité dans le temps long des enquêtes pratiques

2 Lecture mentaliste de l'action contre laquelle Dewey s'est justement opposé (même si il a certainement luimême alimenté cette distorsion dans certains de ses écrits). 
des professionnels, sans nous contenter de leurs seules représentations, mais en élargissant notre observation aux accomplissements concrets produits en réponse aux sollicitations de l'environnement (Mezzena, Seferdjeli \& Stroumza, à paraître). Elle évite donc une hiérarchisation des activités sur les terrains selon la présence ou non de réflexivité.

\section{Le risque d'une définition négative et restreinte de la professionnalité comme effet du paradigme réflexif}

Rappelons la principale thèse de Schön au sujet de la figure du praticien réflexif: les professionnels peuvent se former à une réflexion dans et sur l'action pour dompter l'incertitude de leur pratique et mieux s'autoréguler dans le cours de l'activité (Schön, 1994). Schön a cherché, en s'appuyant à l'origine sur la philosophie de Dewey pour son travail de doctorat (Schön, 1992), à lutter contre une conception «applicationniste » de la connaissance, en rendant justice à la part revenant en propre aux professionnels dans la construction des problèmes. En dépit de ses intentions, il s'est néanmoins maintenu dans une voie intellectualiste : en faisant de la réflexivité des professionnels le «savoir caché dans l'agir », il en fait une intelligence additionnelle à la pratique et de même nature que le savoir, si ce n'est qu'elle est cachée et pas encore (ou plus) formalisée. Il présupposerait ainsi que l'action ou l'intelligence de l'action peut être réduite à la pensée des professionnels et accessible par sa formalisation, faisant en dernier lieu de leurs représentations le lieu où se joue le savoir-faire.

Dans ce modèle, l'analyse de leur action par les professionnels est à la source de l'amélioration et du contrôle de l'action : la qualité de la pratique est dépendante de leur réflexivité «dans et sur l'action», entendue comme mise à distance critique et comme explicitation ou formalisation de la pratique, via une démarche analytique ou introspective. Dans ce modèle et ses usages dérivés, l'analyse des idées, représentations, connaissances ou intentions sont à la base de la détermination de l'action, voire son unique source en étant présentées comme des dispositions appartenant aux professionnels et pouvant être «contrôlées» par eux à partir d'un effort de réflexion. En travail social, ces dispositions personnelles sont souvent étendues aux traits de personnalité, renforçant une lecture privatisant le savoir-faire : analyser ses émotions ou ses caractéristiques personnelles est présenté comme une condition sine qua non pour la construction de la professionnalité.

Dans ce «paradigme réflexif» (Recherche et Formation, 2001), le professionnel est donc au centre de l'action, il en est la principale source de détermination via un travail de pensée sur lui-même lui permettant de réguler l'action en cours d'activité, à partir de l'analyse du problème à traiter. D'un côté, le modèle du praticien réflexif a pour effet de valoriser la pratique en reconnaissant aux professionnels une capacité de réfléchir leur pratique pour l'améliorer et l'alimenter, sans que ce travail de réflexion soit le monopole des chercheurs en sciences humaines. De l'autre, ce modèle a pour revers de cantonner les professionnels à une figure de praticiens certes réflexifs, mais néanmoins voués à doter leur action d'analyses ou de théories pour lui donner de la valeur, comme si l'accomplissement de la pratique ne pouvait être en soi le lieu de production de connaissances. Ce modèle tend ainsi à intellectualiser l'action aux dépens d'une intelligence de nature pratique: si on loge l'intelligence d'une activité dans la réflexion uniquement, ne risque-t-on pas alors d'alimenter une définition exclusivement intellectualiste qui nous ferait passer à côté d'un savoir-faire non applicationniste, construisant une connaissance professionnelle dans la pratique même?

Quoique fort séduisant, car valorisant la capacité d'analyse des professionnels (dont on ne doute d'ailleurs pas), ce modèle présente ainsi du point de vue de la professionnalité deux écueils de taille. On aboutit à une définition de la professionnalité qui est négative lorsque l'on a affaire à des échecs, de l'impuissance ou de la perte de maîtrise, ou encore parfois à 
des pratiques tâtonnantes ou d'une efficacité aléatoire. Il remet en effet en question les professionnels eux-mêmes en cas de problèmes persistants: sont-ils assez réflexifs ? Analysent-ils leur pratique de manière pertinente ? Et d'autre part, on nie l'expertise ou les savoir-faire nécessaires à des activités décrites dans le champ professionnel comme étant à la base du métier qui ne sont pas toujours accompagnées d'une réflexion au sens intellectualiste.

\section{Limites de la réflexivité des professionnels pour guider l'activité}

Notre terrain de recherche et son contexte de dédoublement de mission sont particulièrement intéressants pour étudier le rôle ou la place de la réflexivité dans l'activité, du fait de changements institutionnels venant bousculer la pratique. Dans le cas que nous avons pu étudier, l'équipe s'est vue enjointe par l'autorité de protection de la jeunesse, exécutant les directives des politiques publiques, à mettre en place une nouvelle mission d'insertion suite au constat d'un manque de dispositifs d'accompagnement dans la région pour les jeunes en rupture de formation. Il a fallu créer des ateliers d'insertion, avec l'engagement d'un maittre socio-professionnel (MSP). Celui-ci devait développer un atelier d'activités de production aux côtés de quelques éducateurs proposant, de leur côté, des ateliers de développement complémentaires à l'atelier d'insertion. Les jeunes sans activité de formation se sont vus contraints par la direction d'aller quatre jours par semaine aux ateliers et ils ont fortement résisté de diverses manières : refus de se lever le matin, remise en question et négociation systématique des activités proposées, ou encore pour reprendre le terme d'une éducatrice, «sabotage » durant les ateliers. De nouveaux problèmes pratiques n'ont alors pas manqué d'émerger : comment contraindre les jeunes à se lever ? Comment s'assurer qu'ils se rendent bien aux ateliers ? Comment les motiver voire les obliger à participer? Bref, comment rendre légitime l'autorité éducative sans trop contraindre les jeunes et risquer par là de perdre le sens éducatif de la mission (voir par exemple Ion \& Ravon, 2012)?

Malgré l'investissement dans des démarches réflexives diverses (analyses approfondies, notamment en supervision, délibérations longues et répétées, parfois sur plusieurs jours d'affilée, donnant lieu à des décisions collectives et à la rédaction de textes), des difficultés pratiques ont émergé et ont persisté dans le collectif (notamment augmentation des tensions avec les jeunes et difficulté de coordination avec le MSP). Elles ont donné lieu à des critiques, voire à de la suspicion à l'égard de l'équipe de la part de la direction et à un manque de reconnaissance de la part des partenaires institutionnels : si les problèmes ne se résolvent pas, est-ce à dire que les éducateurs manquent de compétences ? Peut-être sont-ils résistants au changement? Parfois même, la critique s'installait entre les éducateurs euxmêmes: le collègue cherche-t-il vraiment à comprendre mes difficultés ? En cas de difficultés massives ou récalcitrantes, l'entrée par la réflexivité pour expliquer le guidage de l'activité aboutit à une professionnalité définie comme négative via la formulation d'un jugement d'incompétence : si malgré toutes ces démarches réflexives, les professionnels ne parviennent pas à résoudre leurs difficultés, ou ne parviennent pas à se mettre d'accord, ou encore n'arrivent pas à faire l'effort de dépasser les tensions, c'est qu'ils ne réfléchissent pas suffisamment ou que leurs analyses manquent de qualité. On présuppose ainsi qu'analyser la pratique et appliquer des idées devrait permettre mécaniquement, à tous les coups, de maîtriser l'activité et son incertitude.

Depuis plusieurs années, divers courants de l'analyse du travail discutent ces présupposés. Les ergonomes ont bien montré que les professionnels font beaucoup plus dans l'activité que ce qu'ils disent depuis leur réflexivité quand on les sollicite pour décrire leur pratique (par exemple Schwartz, 1989). Dans notre empire, ce constat est manifeste dans les écarts entre les activités observées et les discussions lors des colloques qui visent à décrire ce qu'ils font (réunions d'équipe hebdomadaires, colloques exceptionnels sur plusieurs jours pour l'élaboration des concepts pédagogiques du foyer et des ateliers et des fiches de description 
des ateliers, etc.). Schön lui-même reconnaît cet écart entre le dire et le faire, ce qui l'a d'ailleurs initialement motivé à alimenter une «épistémologie de l'agir professionnel » à partir d'une formation des professionnels à la démarche réflexive afin qu'ils puissent formaliser et conceptualiser leur pratique. Cet écart a amené la psychodynamique du travail à reconnaître l'importance du corps dans l'intelligence pratique (Dejours, 1993), et le courant du cours d'action à parler de conscience préréflexive comme présence à soi (Theureau, 2006). Celle-ci est alors considérée comme montrable, racontable, «commentable», «mimable» dans certaines conditions. Et en effet, nous observons que la formalisation de l'action par la réflexivité a ses limites. Si l'on se cantonne à l'explication de l'action depuis leur réflexivité, des pans entiers de l'activité ne sont pas rendus visibles ou s'avèrent inintelligibles: par exemple, la variété des manières de faire, y compris chez un même professionnel, n'est pas d'emblée décrite ou commentée par les professionnels. Comment comprendre que les éducateurs puissent par moment réagir de telle manière et à d'autres complètement différemment? Comment expliquer les variations dans leurs manières de faire sans suggérer un manque de cohérence ou de constance dans le collectif?

\section{Définir le savoir-faire dans l'activité en partenariat avec l'environnement}

Les travaux de la sociologie de l'action d'Ogien et Quéré (2005), et plus largement le courant de l'action située, permettent de trouver une intelligibilité à ces limites de la réflexivité, en les expliquant depuis les propriétés de l'action et non pas des personnes (dans leur capacité d'analyse et de formalisation). L'activité est définie comme se constituant selon un principe de détermination interne depuis le partenariat avec l'environnement. Quéré (2006) désigne ainsi une relation interne entre organisme et environnement et non pas un partenariat au sens externe d'entités préexistantes, indépendantes et que l'activité ne transforme pas. L'activité est ainsi le fruit d'une pluralité de déterminismes - les jeunes, les collègues, les règles institutionnelles, les prescriptions, la hiérarchie et des partenaires institutionnels, mais aussi le dispositif éducatif, l'espace ou les objets, les règles, etc. Les professionnels sont une force parmi d'autres. Différentes forces, dont notamment des idées actualisées par les professionnels, mais pas seulement, participent donc de la constitution de l'activité. Ils doivent faire avec les autres forces en s'y ajustant dans le moment et en essayant de faire tendre malgré tout l'activité vers certains effets en regard de la mission poursuivie. Selon la manière de «répondre » aux conduites des jeunes ou de tout autre objet de l'environnement, et selon les autres forces en jeu - et « répondant » à leur tour -, l'activité prendra un autre cours.

Pour les professionnels il s'agit de travailler de concert avec les autres forces en ne se laissant pas complètement déterminer par elles, car la mission les invite à mettre en œuvre une certaine perspective. Si les forces extérieures aux professionnels sont agissantes sur l'activité, elles sont aussi en retour travaillées par les professionnels qui concrétisent à leur tour une force et transforment l'environnement. L'intelligence pratique des professionnels se loge dans ce jeu de forces avec lesquelles il s'agit de faire en situation, dans le vif de l'activité en train de se dérouler, en s'appuyant sur elles ou en contournant leurs entraves. Et nous constatons que les idées seules ou plus spécifiquement la réflexivité des professionnels n'est pas suffisante pour tenir dans ce jeu des forces.

Selon les situations, la distribution entre les forces varie. Au fil de l'activité, les rapports entre les forces se distribuent différemment selon les variations des objets et leurs ajustements réciproques. Selon la donne du moment dans la situation, qui inclut ce qui a précédé et les horizons de possibles ouverts par cette donne, l'activité n'appellera pas les mêmes manières de s'ajuster de part et d'autre des forces. Une grande variété dans les manières de faire peut ainsi s'observer. De plus, l'effet obtenu suite à un ajustement (un mot, un geste, un regard... ou une position de retrait) ne sera pas forcément obtenu à une autre 
occasion dans une situation apparemment similaire. Un ajustement peut changer le cours de l'activité et les professionnels, quoique familiarisés avec leur pratique, ne peuvent jamais présumer à quels ajustements ils auront affaire en retour et sur quels ajustements tabler pour la suite. Tant chez les professionnels que chez les jeunes, ou chez toute autre force intervenant dans le cours de l'activité, il n'y a pas une seule et bonne manière de s'ajuster. D'où la fréquente réponse « ça dépend », lorsqu'on demande aux professionnels comment ils s'y prennent : cela dépend effectivement des forces en jeu et de leurs conduites dans le moment, en rapport avec ce qui a précédé et ce qu'ils cherchent à faire dans l'instant. Nous avons affaire à la propriété d'incertitude de l'action : chaque ajustement est dès lors en soi une expérimentation, quelque chose est essayé dont l'effet ne peut être complètement maîtrisé.

En outre, les forces sont agissantes de manière changeante et s'influencent entre elles dans le cours de l'activité. Ces propriétés de «dynamicité » et d'ajustement de l'action expliquent que les professionnels ne peuvent rendre compte de la pluralité des déterminations à l'œuvre dans la constitution de l'activité, ainsi que de leur mutualité. Les professionnels peinent à décrire ces incessantes variations intervenant en cours d'activité et auxquelles ils doivent s'ajuster continûment, relevant des conduites des autres objets de l'environnement. Enfin, bien souvent, leurs propres variations de conduite ne font pas l'objet d'un travail réflexif, mais s'appuient sur des habitudes suffisamment incorporées pour ne plus avoir besoin d'y penser consciemment. Il est ainsi difficile pour les professionnels de décrire tout ce dont ils tiennent compte (de surcroît sans y penser forcément), tant les raisons pratiques sont nombreuses, interdépendantes et variables dans le cours de l'action. En fin de compte, la nature processuelle de l'activité empêche les professionnels de décrire de manière généralisée des ajustements dont le fonctionnement est fondamentalement singulier, car situé. Dans la pluralité des ajustements auxquels elle donne lieu, l'activité engage les professionnels dans un «faire-avec» les autres forces qui exige d'eux plus que leur réflexivité : ces forces, en effet, ne se contentent pas d'accueillir passivement les idées des professionnels, elles y réagissent et peuvent opposer résistance en ne se soumettant pas à leur application. Les forces peuvent tirer l'activité dans une autre direction en s'ajustant d'une autre façon que celle attendue. Ces limites de la réflexivité trouvent aussi une intelligibilité dans le travail du corps, prenant appui sur un savoir incorporé justement indicible, car non réfléchi.

En rattachant les limites de la réflexivité des professionnels non pas à leur personne, mais à la nature même de l'action, nous évitons d'attribuer l'inintelligibilité de la pratique à un déficit de réflexivité et évitons par là de renvoyer aux professionnels toute la responsabilité d'éventuels manques dans l'action. En considérant le savoir-faire non pas comme une réflexion au sens intellectualiste, mais comme un «faire-avec» les autres forces, nous pouvons également considérer que des pratiques dites de base qui ne semblent s'accompagner d'aucune réflexion, puissent tout de même être le lieu d'un savoir-faire, d'une expertise.

S'ouvre alors un espace théorique et empirique propice au repérage d'un savoir-faire de nature pratique, qui n'est pas subordonné à la mise en œuvre de connaissances définies en amont et du dehors de l'activité, de manière intellectualiste, mais se déploie dans l'activité même, sans coupure entre pensée et action, dans l'expérimentation des moyens à disposition dans les situations pour construire l'intervention. Les idées des professionnels, qui renvoient notamment à des expériences antérieures, des connaissances théoriques à expérimenter dans l'action ou encore des anticipations de risques, sont sans cesse entremêlées à ce qui se passe dans le cours de l'activité. Elles sont confondues dynamiquement dans des observations, des gestes et des ressentis, et enchâssées dans les conduites des autres objets. Par ailleurs, ces idées ne sont pas à localiser dans le mental des professionnels, en leur étant attribuées en propre comme s'ils en étaient à la fois l'origine et le lieu de stockage. Elles existent dans le monde, dans la pratique et le champ professionnel, et plus largement dans l'environnement. 
Celles-ci peuvent ainsi être agissantes dans le cours du déroulement de l'action même si le professionnel n'est pas d'accord avec celles-ci (ne partage pas ces idées). Le professionnel n'est donc pas seul aux commandes de l'activité, le guidage opère depuis le partenariat avec l'environnement. L'enjeu du point de vue du savoir-faire est bien de parvenir à influencer ce partenariat pour l'orienter dans le sens de la mission, en redéfinissant sans cesse les problèmes (leur état) en fonction de ce qui se passe concrètement dans le moment et des ressources de la situation à partir des conduites des autres forces (Mezzena \& Stroumza, $2012 \mathrm{a} ; 2012 \mathrm{~b})$.

\section{Avec Dewey : des enquêtes pratiques pour construire des problèmes dans des successions d'agencements}

Ces constats nous ont amenés, toujours à partir de l'examen détaillé des accomplissements des professionnels, à opérationnaliser la notion d'enquête pratique de la philosophie pragmatiste de Dewey (1938/1993). Malgré l'incertitude et l'indexicalité des activités, l'enjeu pour la professionnalité consiste à déterminer ce qui guide l'activité et comment, pour que celle-ci réponde à sa mission. La notion d'enquête pratique couplée à celle d'agencement et de perspective nous permet de décrire ce guidage. Le collectif des professionnels peut être considéré comme un organisme en quête d'équilibre dans ses rapports avec son environnement. Ses besoins le poussent à explorer les potentialités de ce dernier pour résoudre ce qui est trop indéterminé, en expérimentant de nouvelles relations avec lui et en construisant des problèmes sur lesquels opérer. L'observation de la constitution de l'activité dans le temps même de son déroulement rend visible combien l'action ne se satisfait pas d'une logique faisant d'elle le lieu d'application d'idées, d'analyses ou de théories ${ }^{3}$ et exige des enquêtes des professionnels pour construire les problèmes pratiques.

En travaillant à la définition pratique d'un problème (Mezzena, à paraître), les éducateurs expérimentent des voies d'action qui vont à leur tour faire entrevoir d'autres aspects problématiques insoupçonnés jusque-là, ou alors des aspects familiers, mais dont l'actualisation n'est pas prévisible. La manière dont les problèmes vont se poser concrètement au fil des ajustements pour cette situation précise, dans cette enquête-là, ainsi que les voies à emprunter pour les définir dans le détail de leur accomplissement pratique ne peuvent être complètement anticipées. Une connaissance actualisée comme moyen d'agir dans une situation (par exemple tenir compte du fait qu'un adolescent peut avoir l'esprit de contradiction) ne sera plus forcément une ressource efficace dans une autre. La construction des problèmes pratiques relèvent ainsi d'expérimentations, et notamment (mais pas seulement) d'expérimentations de connaissances et d'idées dont il s'agit d'apprécier les effets à partir des ajustements qu'offrent en retour les autres objets de l'environnement. Comme les problèmes ne surgissent pas en étant déjà tout construits, les expérimentations sont nécessaires tant pour la définition que pour la résolution des problèmes pratiques. Des variations dans le cours des choses peuvent remettre en question les balises pratiques préalablement posées lors d'un premier processus expérimental ou lors d'autres enquêtes. Les enquêtes visent à changer les conditions de la situation pour influencer la constitution du problème et tenir dans l'activité en l'orientant dans le sens de la mission.

La notion d'enquête pratique, associée à celle d'agencement, telle que de Jonckheere (2010) et Quéré (2006) la définissent à la suite de Deleuze et Parnet (1996), a l'avantage théorique de pouvoir tenir compte de la «dynamicité » et de la singularité des conditions réelles à

Là encore, Schön était initialement en accord avec cette affirmation qui était à l'origine de sa réflexion. Néanmoins, ne renonçant pas au rôle des représentations, il reste dans une conception applicationniste, faisant de l'action le lieu d'application d'idées travaillées «dans la tête » des professionnels (Friedrich, 2009). 
l'œuvre dans l'activité. Elle nous aide aussi pour le repérage de régularités portant des habitudes d'action. L'activité peut en effet être décrite, depuis la mutualité des rapports constitutifs entre les objets issus de l'environnement, comme des agencements dans lesquels les forces agissantes tiennent ensemble pour produire l'activité. L'agencement peut être défini comme le fruit des ajustements entre les objets de l'environnement. En laissant la main aux propriétés de l'action, la notion d'agencement revisite la définition de l'engagement des professionnels dans l'activité : elle permet de saisir comment le professionnel est, dans l'agencement, construit par la situation et réciproquement. À la notion de dialectique, qui met ensemble en les rendant dépendantes deux entités opposées, nous préférons la notion d'immanence pour caractériser les agencements : les professionnels se trouvent pris aux côtés d'autres forces dans des rapports mutuels qui ensemble vont déterminer solidairement la constitution de l'activité, dans le temps même de son déroulement. L'agencement produit sa propre organisation de l'intérieur de son déroulement, en fonction de ce qui survient et qui est intégré progressivement dans le cours de l'activité. Et effectivement les professionnels n'ont pas le choix : tout ce qui survient doit être « traité » et exige des ajustements, quitte à faire le choix de ne pas en tenir compte (ce qui déterminera l'activité en creux). Ce sont donc ces agencements qui déterminent la manière dont les professionnels sont engagés dans l'activité, même si en même temps ces derniers sont agissants comme une force parmi d'autres dans la constitution de l'activité (Mezzena, 2012 ; Stroumza, Mezzena, S., Friedrich, \& Seferdjeli, 2013).

\section{Un exemple d'enquête pratique}

Les activités apparemment banales n'échappent pas aux enquêtes : l'activité n'est jamais pré-donnée, il faut la construire à chaque instant, de concert avec l'environnement. Les enquêtes pratiques ne sont donc pas réservées aux problèmes extraordinaires. Prenons l'exemple d'une situation dans laquelle une éducatrice doit amener un jeune à ranger sa chambre. Ce problème est un des problèmes parmi d'autres qui est mis au travail dans l'accompagnement éducatif de ce jeune-là, et les éducateurs connaissent sa difficulté à s'y mettre. L'éducatrice vient lui demander de quitter l'ordinateur sur lequel il est en train de passer du temps dans la salle commune et de monter ranger sa chambre. Lorsqu'elle interpelle le jeune, ce dernier ne fait pas mine d'éteindre l'ordinateur. N'obtenant pas d'effet, elle tente alors de lui rappeler l'engagement qu'il a pris avec son éducatrice référente, à savoir respecter la règle du rangement hebdomadaire de la chambre et la ranger dans le courant de la semaine. Elle lui rappelle également qu'avec un autre éducateur en service ce jour-là, il a passé un accord consistant à s'y mettre pas trop tard dans la soirée. Or il est plus de 21 heures. Le jeune s'ajuste alors en niant l'accord passé avec l'éducateur présent et en remettant en question, mais sans pour autant vouloir en discuter, l'engagement pris avec son éducatrice référente en qualifiant cette décision d' «unilatérale ». Ce faisant, l'éducatrice ne peut poursuivre en optant pour la piste de la négociation. À partir de l'appréciation de ces effets, elle va alors prendre une autre voie consistant à imposer sa présence silencieuse en se tenant sans bouger derrière le jeune, jusqu'à qu'il se mette finalement en route.

L'observation détaillée du déroulement de l'activité pointe les forces auxquelles la professionnelle doit donner le change, tout en essayant de faire prendre une certaine direction à ce qui est en train de se passer. Outre la conduite du jeune, d'autres forces sont agissantes. L'organisation du travail en relais oblige l'éducatrice à poursuivre le travail effectué en amont par ses collègues et «à ne pas lâcher». De même, les connaissances qu'elle a de ce jeune l'amènent à demeurer ferme, mais sans opposition verbale frontale : elle le définit comme pouvant faire de grosses colères et il s'agit de le contraindre à se mettre en mouvement tout en évitant dans la mesure du possible de s'orienter vers un conflit peu fructueux du point de vue éducatif. De plus, en auto-confrontation lorsqu'elle commentera le film de son activité, la professionnelle précisera que la façon dont elle opère dans ce moment 
précis est également induite par le fait qu'elle n'aime pas exiger des jeunes qu'ils répondent séance tenante aux demandes qu'on leur adresse : il s'agit aussi de laisser le temps au jeune de quitter ce qu'il est en train de faire. Ou encore, une fois au bureau après son échange avec le jeune, elle expliquera à son collègue que la règle qui autorise les jeunes à être sur l'ordinateur jusque tard en soirée ne les aide pas à se prendre en main, ainsi le dispositif éducatif est également agissant à cet instant.

Ce «dépliage» non exhaustif des forces intervenant dans cet agencement rend visible la façon dont l'activité se construit processuellement, d'un ajustement à l'autre. D'une seconde à l'autre, l'éducatrice répond d'une certaine manière au jeune et réciproquement, leurs ajustements réciproques s'enchâssant les uns dans les autres tout en étant par ailleurs emboîtés à d'autres forces encore, pour certaines moins tangibles (règles institutionnelles du lieu, moment où se déroule l'action, actions effectuées en amont par les autres collègues dans le travail en relais, connaissances des éducateurs à propos du jeune). L'intelligibilité de ce qui se passe se construit au fur et à mesure en n'étant pas prédéfinie en amont de l'activité, mais bien au fur et à mesure de ce qui s'expérimente dans l'agencement de forces et des effets produits (par exemple depuis les questions posées et les observations du jeune sur le moment qui permettent à l'éducatrice d'apprécier son état et d'anticiper les suites possibles). Ainsi, même dans une activité a priori assez banale, les professionnels doivent enquêter, expérimenter dans le moment pour savoir à quoi ils ont à faire et poursuivre la construction du problème pratique en essayant de changer les conditions de la situation pour le faire avancer dans une certaine direction. Pourtant, aux dires des éducateurs, ce travail d'enquête n'est, dans l'instant, quasiment pas réfléchi. De plus, les ajustements incessants ainsi que les expérimentations et leurs appréciations ne sont que très partiellement décrits en autoconfrontation.

Dans l'agencement, rien n'est opposé (ou « dialectisé »), mais tout y est intégré comme «un tout contextuel » (Dewey, 1938/1993), comme « un tout complexe en soi, individualisé par sa qualité diffuse, ainsi que dans l'interaction entre l'organisme et l'environnement » (Quéré, 2002, p. 136). Cette définition de l'agencement comme synonyme de situation nous amène à la question de la perception. L'action n'est pas conçue comme un contenu qui existerait à l'extérieur des professionnels, dans la réalité, et qui serait prêt à être perçu en étant déjà constitué. La constitution de l'activité opère depuis les ajustements entre les différentes forces et leur agencement, et les rapports qui les constituent sont perçus au moment même où ils s'actualisent. Il n'est donc pas question de problèmes déjà «tout» construits et à percevoir, qui se constitueraient sans le concours du professionnel, dans l'extérieur de l'action et qu'il percevrait ensuite via un travail mental réflexif. Ce sont des problèmes en mouvement et dont la constitution et l'évolution se perçoivent au moment même où ils se constituent dans des successions d'agencements en partenariat avec l'environnement. Notre approche s'inscrit dans la tradition de la cognition distribuée qui va à l'encontre de «la possibilité de séparer la cognition de la perception et de l'action » (Theureau, 2004, p. 14).

La notion d'agencement permet de tenir compte des régularités amenant une certaine stabilité dans l'activité, en la rattachant aux relations entre les objets plutôt qu'aux objets pris isolément. Le savoir-faire des professionnels, constitué localement depuis les expériences des enquêtes menées en partenariat avec l'environnement, acquiert une certaine permanence, à rattacher aux enchaînements d'agencements plutôt qu'à la réflexivité des professionnels. La façon dont les agencements se succèdent, en s'intégrant à leur suite, donne un certain contenu ou teneur à l'activité et produit une certaine manière de construire les problèmes. Des manières spécifiques de construire des problèmes, produites par des successions d'agencements, sont repérables, car récurrentes dans l'observation continue des enquêtes. Elles sont le fruit d'une pratique qui s'est instituée progressivement, en «apprenant » aux objets à s'ajuster et à fonctionner ensemble malgré les incessantes variations de leurs rapports mutuels. Mais il ne s'agit pas d'une simple répétition mécanique pour autant: il faut un «travail», un effort pour que les agencements existent, s'actualisent. De la même 
manière, les agencements ne s'actualisent ni par magie ou par volonté réflexive, de sorte que tenir dans leur succession, en tentant de les maintenir ou de les transformer et de les orienter dans une certaine direction, exige aussi d'expérimenter des voies d'action dans le moment même, aux risques et périls de la mission. Ainsi, les constructions de problèmes inhérentes aux enchaînements d'agencements sont toujours susceptibles d'évoluer dans le temps long des enquêtes. Dès lors, les habitudes ne sont pas rattachées aux seuls professionnels, mais sont attribuées aux rapports mutuels entre les objets de l'environnement et à leur dynamique, dont le sort dépend du partenariat avec l'environnement et non pas des seuls professionnels.

\section{Enquêtes pratiques et perspective}

Dans les enquêtes, les problèmes mutent sans cesse (Mezzena, à paraître). D'une situation à l'autre, les termes du problème changent, ce qui exige des professionnels de le redéfinir d'une fois à l'autre en expérimentant pour apprécier ses nouvelles conditions et tenter de les transformer pour faire avancer le problème. L'analyse d'activités nous a permis d'étayer empiriquement les notions d'expérimentation, d'anticipation, d'appréciation à l'œuvre dans les enquêtes pratiques des professionnels. Nous avons également travaillé la notion de perspective permettant de saisir comment les professionnels parviennent à tenir le cap de leur mission dans l'activité, malgré l'incertitude de l'action et les ajustements situés et dynamiques avec lesquels ils doivent sans cesse composer. La perspective peut se définir comme la visée générale à tenir, mais sans cesse à redéfinir pratiquement, en faisant à partir de, et avec les conditions réelles des situations et de l'étape de l'enquête en cours. Dans les enquêtes pratiques, selon les forces en jeu les moyens sont revisités en cours de route. L'activité est donc déterminée depuis l'aval de son déroulement: des fins intermédiaires sont progressivement déterminées à partir des ressources de la situation; ressources qui émergent dans le cours de l'activité, sont appréciées dans l'instant et deviennent momentanément et pour cette situation-là des moyens d'agir. L'intentionnalité est donc distribuée sur les rapports mutuels entre forces, dans le système téléologique que forment l'organisme et l'environnement (Quéré, 2006) et non pas dans une pensée (au sens de représentations en termes de buts ou d'objectifs) qui serait attribuée aux seuls professionnels.

Cette logique moyens-fins permet aux professionnels de toujours polariser l'activité dans la même direction - la perspective générale de la mission - tout en faisant avec les conditions réelles toujours singulières et par définition différenciées - une perspective locale - auxquels ils ont affaire au fil des situations dans la construction de leurs problèmes pratiques. Dans la mise en ouvre pratique de la mission, la perspective fait tenir aux professionnels un certain cap tout en intégrant dynamiquement, au fil de la variabilité des situations, une pluralité de forces renvoyant à une multitude d'enjeux tirant dans des directions différentes. Le savoirfaire des professionnels consiste à donner une orientation à l'activité en intégrant cette pluralité de mouvements. Il s'agit de trouver un équilibre dans la succession et dans la pluralité des problèmes qui s'y construisent. Les pistes pratiques sont appréciées dans l'instant, non pas abstraitement à partir de l'évaluation d'unités discrètes, mais de manière sensible, en rapport avec une globalité qui a à voir avec les enquêtes antérieures et leurs effets pour la poursuite de la perspective. Des conséquences concrètes, sous forme de scénarios pratiques probables, sont envisagées et des risques, dont les conséquences pourraient menacer la pratique en faisant sortir l'activité de la mission, sont sans cesse anticipés, même s'ils ne peuvent pas être complètement évités. Ainsi les frontières de la mission ne sont pas prédéfinies et établies une fois pour toutes : elles sont continûment appréciées et retravaillées, déplacées depuis la manière spécifique dont les problèmes se construisent progressivement, et l'enjeu central consiste à ne pas s'installer durablement dans certains risques.

C'est dans les détails pratiques que se travaille la poursuite de la perspective de la mission et que se construit un pouvoir d'agir. Des décisions sont éprouvées qui portent sur des micro- 
détails de l'activité. D'un point de vue méthodologique, une analyse filée, c'est-à-dire continue des problèmes pratiques dans le temps long des enquêtes, s'effectue depuis le croisement d'une pluralité de données (analyse des prescriptions, des activités et des autoconfrontations, observations, entretiens pré et post-activité). Leur comparaison et leur recoupement permettent de reconstruire la façon dont cette équipe travaille la maturation des jeunes, c'est-à-dire entreprennent de favoriser un mouvement sur la durée portée par le jeune, et à quelles manières de construire les problèmes pratiques elle donne lieu (Mezzena, Seferdjeli, \& Stroumza, à paraître).

Pour les éducateurs, identifier des indices quant à l'état des jeunes, ou plutôt à l'état du mouvement, construire des séries d'indices à apprécier au fil des situations, évaluer les effets de l'intervention dans le temps est une activité très importante. Il s'agit bien d'éviter un mouvement qui ne serait porté que par l'éducateur et risquerait dès lors de s'arrêter à la sortie du foyer (à 18 ans). Mais le risque anticipé est également celui d'une absence de mouvement (qui peut conduire à l'oisiveté, la dépression, voire à la situation où les jeunes ne quittent plus leur lit) ou une succession de mouvements désordonnés, sans durée réelle (et qui dès lors n'aboutirait pas à de la maturation chez le jeune). L'important dans cette séquence n'est dès lors pas tant que le jeune obéisse par soumission à l'éducatrice, ni qu'il lui parle correctement, mais qu'il se prenne en main. Il y a ainsi un travail d'appréciation de ce mouvement, d'identification des indices et de la série, qui est rendu particulièrement ardu par le fait que l'équipe fonctionne en relais (présence 24h/24) et que l'activité se déroule souvent hors de la vue des professionnels. La maison du foyer est effectivement sur deux étages et les ateliers se déroulent à distance géographique du foyer. Quoiqu'essentielles aux ajustements des professionnels et à la poursuite de la perspective, ces opérations ne sont pas réfléchies: elles ne sont pas spontanément décrites par les professionnels et elles sont très partiellement décrites à l'occasion des auto-confrontations.

Dans les enquêtes des professionnels nous n'avons donc pas affaire à une logique intellectualiste qui prédéfinit tant les fins que les moyens en amont ou en tout cas du dehors du déroulement de l'activité, ce qui laisserait entendre que ses tenants et aboutissants peuvent être anticipés et contrôlés depuis une pensée réflexive. La perspective n'est donc pas réductible à des prescriptions, des objectifs ou des décisions, elle se construit dans l'expérience de l'activité. La perspective est la définition foncièrement pratique de la mission. Au fur et à mesure de leur expérience, et des territoires pratiques que les professionnels sont amenés à sillonner au fil de leurs enquêtes, la manière dont la mission peut être mise en œuvre se (re)dessine progressivement.

\section{Externalisation de la pensée dans l'action}

Les processus d'enquête pratique en partenariat avec l'environnement ne se limitent pas à l'usage d'idées, mais s'appuient sur des observations et des impressions, ce tout indistinct formant un sentir dans l'immanence de l'activité. Ainsi le terme «apprécier » n'est pas à prendre en un sens réflexif, car il ne s'agit pas d'un travail intellectuel.

Cette non-réflexivité dans le déroulement de l'action ne signifie pas que le professionnel ne peut pas les formaliser en situation d'auto-confrontation lorsqu'on le sollicite à l'aide d'un dispositif d'observation indirecte. Ce qui produit de l'intelligence se trouve dans la pratique même et les relations mutuelles et dynamiques entre les forces, à laquelle certes les idées des professionnelles participent, mais qui ne sont pas suffisantes, et sont incessamment affectées en retour par les ajustements des autres forces (et ne sont donc pas seules «à décider»). C'est donc le problème de la définition du rapport entre pensée et action qui est posé. Nos travaux l'abordent depuis la thèse de l'externalisation de la pensée dans la suite des travaux sur la cognition distribuée, avec cette idée que la pensée n'est pas celle d'un sujet, délimitée de manière privée à sa personne et à son mental, comme s'il pilotait "dans sa tête » depuis des représentations opératoires et depuis une position de surplomb à l'égard de ce qui se passe en dehors de lui dans l'activité. La pensée est étendue à, et est logée dans, l'activité : « la cognition ne se situe pas dans la tête, mais dans un entre-deux, entre l'acteur et la 
situation» (Theureau, 2004, p. 14). Nous reconnaissons dès lors l'existence d'un raisonnement pratique, «(..) tel qu'il se constitue dans la temporalité même de son déploiement, c'est-à-dire comme un enchâssement continu d'appréciations instantanées au sujet de "ce qui se passe" (...) ce qui ne revient pas à rapporter cette suite à une délibération ou un calcul au terme desquelles un individu déciderait d'agir d'une manière déterminée » (Ogien, 2009, p. 111).

Est ainsi remise en question l'affirmation d'une pensée synonyme d'une conscience comme condition pour l'action via une réflexivité comme mise à distance et formalisation de la pratique. Entre les deux pôles traditionnellement opposés de conscience et de nonconscience dans l'action, une voie intermédiaire déjà ouverte par Friedrich (2009) est intéressante à creuser : les professionnels ne se disent pas explicitement à eux-mêmes ce qu'ils sont en train de suivre comme raisonnements pratiques. Quoique non réflexifs, ces raisonnements pratiques n'en sont pas moins agissants et leur existence peut être rendue visible a posteriori de l'action. Nous avons affaire à une certaine forme de conscience qui n'est pas seulement celle des situations comme état à un moment donné d'un ensemble de rapports mutuels de forces requérant une intelligence ajustée à cet agencement. Elle est aussi une conscience du mouvement ou de la sérialité : les professionnels sentent dans la succession des agencements et dans la continuité des enquêtes comment évoluent les problèmes pratiques. Leur appréciation opère dans les enchaînements d'agencements que l'activité leur fait traverser. C'est aussi cette continuité qui permet aux professionnels de sentir ex post que la donne change et que les situations ne sont plus les mêmes, car c'est dans le mouvement ou les séries que l'on identifie leur délimitation: «Les frontières d'une situation sont certes floues et changeantes, mais celle-ci n'en a pas moins les caractères de clôture, déterminée par la modification d'interactions préexistantes et par la nécessité de réorganiser les conduites » (Garreta, 1999, p. 38).

\section{Conclusion}

Les enquêtes pratiques ne relèvent pas uniquement d'un travail mental au sens intellectuel qu'on lui prête traditionnellement. Nous avons souligné combien les expérimentations sans cesse à l'œuvre dans l'activité depuis les ajustements, tout comme celle des habitudes avec lesquelles elles cohabitent inextricablement, échappent à la réflexivité des professionnels.

Le savoir-faire déployé dans les enquêtes pratiques en partenariat avec l'environnement ne peut être réduit à une logique applicationniste. Dans celle-ci, la connaissance est définie comme des contenus fixes et permanents stockés à l'intérieur du mental qui peuvent être des idées (conceptions, intentions, théories, pistes ou conclusion d'analyses, ou encore valeurs) ou des descriptions abstraites de manières de faire. Supposées justes en regard des caractéristiques du problème identifié (analysé réflexivement) au préalable, ces représentations opératoires sont ensuite appliquées dans la situation pour agir. La pratique est alors présentée comme le lieu d'application d'idées qui seraient à saisir en dehors de son déroulement, et les professionnels comme les exécutants (certes réflexifs) d'idées ou de théories à aller chercher ailleurs que dans le déroulement effectif de l'activité : dans la réflexion sur la pratique ou dans les connaissances proposées par les théories, impliquant une posture réflexive des professionnels dans le guidage de l'action depuis une position d'extériorité et de surplomb. Cette conception de la connaissance, fondée sur un dualisme entre pensée et action, présuppose que la pratique ne se suffit pas à elle-même pour être le lieu de production de connaissances (Friedrich, 2009). Il s'agit d'une conception représentationaliste mettant en correspondance d'un côté des contenus mentaux (idées, représentations, conceptions, connaissances, mais aussi selon les versions, des valeurs, de la volonté ou traits de personnalité) attribués en propre et de manière permanente à l'intériorité des professionnels, et de l'autre des situations ou des problèmes dans la réalité extérieure de la pratique. D'après cette définition intellectualiste de la connaissance, fondée sur une coupure entre pensée et action, l'intervention sociale renvoie à un sens prédéfini qui préexiste à la mise en auvre de l'action, comme si la pratique venait révéler une 
connaissance transcendante qu'elle exprimerait ou actualiserait dans un réel statique et docile.

Cette recherche remet en question l'univocité de la définition de la professionnalité issue du modèle du praticien réflexif de Schön ou de ses dérivés, qui cantonne le développement professionnel et sa plus-value à la réflexivité des professionnels définie comme travail d'analyse et de formalisation de leur pratique. Ce faisant elle évite d'alimenter et de favoriser une définition négative de la professionnalité en cas de difficultés ainsi que le déni de l'expertise à l'œuvre dans les activités d'accompagnement éducatif au quotidien considérées comme des pratiques de base. L'observation des enquêtes pratiques nous amène plutôt à souligner qu'il ne s'agit pas tant de «faire juste » en regard d'une connaissance prédéfinie et à appliquer que de parvenir à construire des problèmes pratiques en faisant avec les autres objets de l'environnement en expérimentant des relations avec eux. Il s'agit de trouver un équilibre dans les manières plurielles et dynamiques de construire les problèmes qui permettent de fonctionner avec ces autres objets, sans les exclure, et en étant sensibles aux risques qui peuvent menacer la poursuite de la mission. Les enquêtes pratiques des professionnels, fil rouge de ce partenariat avec l'environnement, sont ainsi le lieu de constitution d'un savoir-faire expérimental, et l'activité le lieu même où se construit une connaissance de nature pratique et sensible.

\section{BIBLIOGRAPHIE}

Couturier, Y. (2000). L'inflation réflexive dans le courant praxéologique : indice de la reconstruction de l'idéologie professionnaliste. Nouvelles pratiques sociales, 13(1), 137-152.

Dejours, Ch. (1993). Intelligence pratique et sagesse pratique : deux dimensions méconnues du travail réel. Éducation Permanente, 116, 47-70.

Deleuze, G. \& Parnet, C. (1996). Dialogues. Paris: Champs-Flammarion.

Dewey, J. (1938/1993). Logique. La théorie de l'enquête. Paris: PUF.

Friedrich, J. (2009). Savoir-faire et réflexion. Conférence audio à la journée scientifique du réseau analyse de l'activité de la HETS consacrée à la réflexivité, 9 mars.

Garreta, G. (1999). Situation et objectivité. Activité et émergence des objets dans le pragmatisme de Dewey et Mead. In M. de Fornel \& L. Quéré (Eds.), La logique des situations (pp 35-68). Paris: Éditions de l'EHESS («Raisons Pratiques », 10).

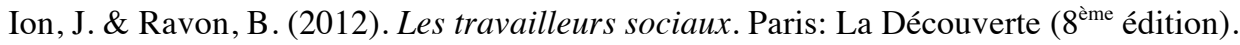

Jonckheere de, C. (2010). 83 mots pour penser l'intervention en travail social. Genève: Éditions Ies.

Mezzena, S. (2011). La réflexivité dans et sur l'action : enjeux pour la professionnalisation des travailleurs sociaux. Revue suisse de travail social, 10(1), 70-83.

Mezzena, S. (2012). Engagement les travailleurs sociaux depuis le partenariat avec l'environnement : agencements et formation des valeurs. Revue Forum, 136, 37-46.

Mezzena, S. (à paraitre). Filage d'une enquête pratique en éducation spécialisée. Revue AFFUTS.

Mezzena, S., Seferdjeli, L., \& Stroumza, K. (à paraitre). Des recherches qui prennent pour objet les savoir-faire. CERTS.

Mezzena, S. \& Stroumza, K. (2012a). Des enquêtes pratiques à la connaissance dans les accomplissements : place des éducateurs, des chercheurs et des théories dans une recherche en éducation spécialisée. Revue Pensée Plurielle, 30-31, 175-189.

Mezzena, S. \& Stroumza, K. (2012b). Des idées agissantes dans l'activité : analyses d'enquêtes dans l'activité réelle d'éducateurs spécialisés. Revue DIRE, 2.

http://epublications.unilim.fr/revues/dire/171.

Ogien, A. (2009). Le non-lieu de l'erreur. In C. Chauviré, A. Ogien, \& L. Quéré (dir.), Dynamiques de l'erreur (pp. 111-131). Paris: Éditions de l'EHESS («Raisons Pratiques», 19).

Ogien, A., \& Quéré, L. (2005). Le vocabulaire de la sociologie de l'action. Paris: Ellipses.

Quéré, L. (2002). La structure de l'expérience publique d'un point de vue pragmatiste. In D. Cefaï \& I. Joseph (Eds.), L'héritage du pragmatisme. Conflits d'urbanité et épreuves de civisme (pp. 131160). Paris: Edition de l'Aube. 
Quéré, L. (2006). L'environnement comme partenaire. In J.-M. Barbier \& M. Durand (Eds.), Sujets, activités, environnements. Approches transverses (pp. 7-29). Paris: PUF.

Recherche \& Formation (2001). «Le praticien réflexif. La diffusion d'un modèle de formation », 36.

Schön, D. A. (1992). The theory of Inquiry : Dewey’s Legacy to Education. Curriculum Inquiry, 22(2), 119-139.

Schön, D. A. (1994). Le praticien réflexif. À la recherche du savoir caché dans l'agir professionnel. Montréal: Les Éditions logiques.

Schwartz Y. (1989). «C'est compliqué ». Activité symbolique et activité industrieuse. Langages, 93, 98-109.

Stroumza, K, Mezzena, S., Friedrich, J., \& Seferdjeli L. (2013). L'ajustement dans tous ses états : règles, émotions, distance et engagement dans les activités éducatives d'un centre de jour genevois. Genève: Editions ies.

Tardif, M., Borges, C., \& Malo, A. (Eds.) (2012). Le virage réflexif en éducation. Où en sommes-nous 30 ans après Schön? Paris: De Boeck.

Theureau, J. (2004). L'hypothèse de la cognition (ou action) située et la tradition d'analyse du travail de l'ergonomie de langue française. @ ctivités, 1(2), 11-25.

Theureau, J. (2006). Le cours d'action. Méthode développée. Toulouse: Octarès.

\begin{abstract}
RESUME
Depuis l'approche située de la sociologie française de l'action de Quéré et Ogien, articulée à la philosophie pragmatiste deweyenne, nos analyses de l'activité d'éducateurs spécialisés ainsi qu'une littérature plus large en analyse du travail mettent en évidence que la réflexivité des professionnels n'est pas suffisante pour rendre compte de la construction de l'intervention et de son intelligence. Définie par le modèle du praticien réflexif de Schön et ses dérivés comme un travail de mise à distance, d'analyse et de formalisation de leur pratique, elle alimente une conception négative et restreinte de la professionnalité. Notre recherche s'est déroulée dans une structure d'hébergement pour adolescents en rupture familiale qui se voit enjoindre par le politique la mise en place d'une nouvelle mission d'insertion. Malgré de nombreuses réunions et discussions informelles, l'activité échappe sans cesse à une formalisation. Depuis un modèle alternatif de professionnalité basé sur la notion d'enquête deweyenne, nos analyses des enquêtes pratiques des professionnels, à la fois immanentes et filées dans le temps, montrent que la construction de l'intervention exige un savoir-faire logé dans l'activité même, et ce même pour des pratiques dites de base. Elle exige incessamment de par les propriétés de l'action l'expérimentation d'ajustements qui font que la mise en œuvre de la mission ne relève pas d'une logique applicationniste. Cette approche conduit à une forme d'externalisation de la pensée dans l'activité, définissant la pratique comme le lieu même où se construit la connaissance, sans la subordonner à la réflexivité comme intelligence de nature intellectuelle additionnelle à la pratique.
\end{abstract}

\title{
MOTS CLES
}

activité, enquête pratique, agencement, perspective, externalisation de la pensée

\section{REFERENCEMENT}

Mezzena, S., Stroumsza, K., Seferdjeli, L., \& Baumgartner, P. (2013). De la réflexivité du sujet aux enquêtes pratiques dans l'activité d'éducateurs spécialisés. Activités, 10(2), 193206, http://www .activites.org/v10n2/v10n2.pdf

Article soumis le 5 février 2013, accepté pour publication le 23 juillet 2013 